\title{
COVID-19 and Beyond: A Prototype for Remote/Virtual Social Work Field Placement
}

\author{
Barbara Mitchell ${ }^{1} \cdot$ David Sarfati $^{1}\left[\right.$. Melissa Stewart ${ }^{1}$
}

Accepted: 9 January 2021 / Published online: 4 February 2021

(c) The Author(s), under exclusive licence to Springer Science+Business Media, LLC part of Springer Nature 2021

\begin{abstract}
The COVID-19 pandemic necessitated an abrupt conclusion of field placement for social work interns at a comprehensive cancer center. In response to social distancing requirements, social workers, but not interns, were granted access to work remotely. Virtual programming became necessary to meet the interns' remaining educational requirements and provided an opportunity for proper termination from the program. This article will delineate the program redesign for oncology social work interns using remote/virtual modalities. This melded approach involved creating simulated clinical interactions, based on selected points along the illness trajectory targeting specific clinical competencies, which were presented to interns by phone and/or videoconference. Examples will be provided related to developing clinical skills and critical thinking and preparing for professional responsibilities within a broad range of healthcare settings. Guidelines for working with individuals, couples/families, and groups will be included. Issues of individual and group supervision will be explored, with sensitivity to the parallel experience of existential uncertainty and mortality awareness among the interns in the context of the pandemic. Although in-person training is preferable, there are advantages to virtual learning for both supervisors and interns. This creative adaptation of field education provides an innovative programming model that can be used to enhance the experience for social work interns moving forward in various healthcare settings during ordinary or extraordinary circumstances.
\end{abstract}

Keywords Clinical training $\cdot$ Virtual/remote $\cdot$ Field $\cdot$ Internship $\cdot$ Medical/oncology social work

In early spring of 2020, the COVID-19 virus swept through New York City and necessitated an abrupt conclusion of field placement for social work interns at a major cancer center. Manhattan and the surrounding boroughs sat at the epicenter of the pandemic that spread throughout New York State and parts of New Jersey. In efforts to flatten the curve (Hogan et al. 2020), shelter-in-place guidelines were issued by the Governor of New York. The institution was mobilized to care for an influx of patients with COVID-19 and inpatient units were reconfigured to accommodate them. In the outpatient setting, social distancing requirements led to the postponement or rescheduling of clinic visits and cancer treatments were delayed, staggered and, in some cases, cancelled entirely. The cancer center's visitor policy also was restricted to prohibit the spread of the virus; no visitors were permitted on-site with very few exceptions. All social

Barbara Mitchell

mitchelb@mskcc.org

1 Memorial Sloan Kettering Cancer Center, New York, NY, USA workers were quickly transitioned to remote work, even as they mobilized to respond to the intense psychological need of patients, family members and interdisciplinary colleagues. Educational institutions, in a need to protect their students and faculty cancelled in-person classes and suspended field placements, while determining next steps based on rapidly shifting CDC and local government guidelines (CDC: Centers for Disease Control and Prevention 2020). These adjustments were made continuously over the difficult days and weeks of the initial surge.

Schools of social work coordinated their program requirements with the Council on Social Work Education (CSWE and Commission on Accreditation Statement, May 9, 2020). When classes resumed, they were offered only online. Field placements for many interns shifted to remote work or in some cases were cancelled entirely. Due to concerns about privacy and security of personal health information in a nonclinical setting (Department of Health and Human Services 2020), social work interns at this cancer center were not granted the same remote access as staff to view electronic medical records from their personal desktops. Under these 
circumstances, the educational program for interns could have been completed early; however, it was decided to provide clinical education and supervision in a modified virtual/ remote capacity.

The social work internship was offered to full-time students in the second year of their Masters' degree training. Traditionally, interns were expected to be on-site, primarily in either an inpatient unit or outpatient clinic. The internship program included 1-hour didactic training on various topics related to clinical oncology social work in a medical setting. In addition, each student was required to attend 1-hour of group supervision and a minimum of 1-hour of individual supervision per week. When the pandemic hit the city, inperson field placement was suspended by the educational institutions, and within the hospital the transition to remote patient work began. Remote work refers to the ability to contact patients by phone or videoconference. Remote work also refers to the ability of staff to connect with secure hospital information systems while off site. This type of access requires secure devices and environments that could not be adequately arranged for interns. However, virtual learning, observation of online open support groups, and supervision, by phone and/or videoconference, could continue. Unfortunately, interns would no longer have access to patients, their medical records or family caregivers. This presented a challenge and an opportunity to shift to a virtual format for the remainder of the semester. In this extraordinary circumstance, the pandemic served as a catalyst for implementing a "patient-less" clinical experience. The model described in this paper can be applied to other scenarios where it may be appropriate to develop clinical skills in the absence of patients, such as when interns are not yet ready for direct contact with patient/clients or if there is a period of low patient/client volume in a particular setting. It is important to note that this abrupt transition to a virtual learning model matched the growing demand by oncology patients for telemedicine which could potentially ease the burden of receiving medical care.

Although graduate schools adjusted the hours required to meet the standards for successful completion of the Masters' degree program from 600 to 450 hours/year (CSWE 2020), virtual programming became an option to meet interns' remaining clinical educational requirements. One obvious challenge was that individualized needs would have to be taken into consideration as not all interns were at the same level in their individual learning trajectory, and some required more opportunities to develop their clinical skills before entering the workforce. The existence of the pandemic itself provided, and continues to provide, a very personal insight into the cancer patient's experience of uncertainty, loss of control, and the awareness of one's own mortality. Interns and staff became sensitive to this poignant parallel experience and its relevance to oncology social work. More time and attention were needed to process anticipatory grief and termination of the clinical relationship between interns and their cancer patients, supervisors and peers. Finally, the program was continued because it was important to provide connection, continuity, and emotional support in response to the pandemic for both interns and supervisors, all of whom required some level of stabilization and consistency during the crisis.

\section{Methods}

\section{Adjustment to the Program Structure in Response to the Pandemic}

The social work internship redesign in response to the pandemic and the ensuing shelter-in-place guidelines included weekly social work staff presentations offered by phone and/ or videoconference. The training for the remaining 5 weeks was focused on further developing interns' clinical skills and critical thinking abilities, as well as preparing them for professional responsibilities within the medical setting.

In order to provide virtual clinical experiences for interns, patient scenarios were carefully selected to highlight CSWE core competencies, specifically engaging, assessing and intervening with individuals, families, groups, organizations, and communities (CSWE 2015). These were presented to simulate clinical interactions through role-playing with the supervisors. The supervisors discussed and modelled multidimensional professional skills and the interns were able to observe and apply them through role-play (Molina et al. 2018). This could be expanded to involve several interns in a more dynamic family, group or team meeting role-playing experience. Interns were also able to observe staff-facilitated online support groups. To prepare them for clinical interactions, books (memoir, fiction) and academic articles were provided to enhance the learning and expand their knowledge base about specific topics. After role-playing interactions, interns were expected to write process recordings, considering their thoughts and emotional responses to the session, and/or reflection papers to further develop critical thinking skills. Reflections and process recordings were reviewed by supervisors who continued to provide feedback and individual supervision and group supervision by phone or videoconference. Interns, now without access to medical records and chart review, were still given the opportunity to practice the documentation style and word-choice most fitting for a legal patient record. Facilitated discussions with interns focused on gaining an understanding of how social workers, other disciplines, and the cancer center were responding to the pandemic. 


\section{Learning Modules}

The content of the modules weaves together three different tracks that cover (1) points along the illness (oncology) trajectory, (2) the role of the social worker within the medical setting and processes related to the medical setting, and (3) clinical issues. Points along the illness trajectory include but are not limited to new diagnosis, recurrence, post treatment/survivorship, end of life, and anticipatory grief and bereavement. New diagnoses span the following options: a favorable prognosis, treatable, likely curable, or a chronic condition, treatable, but incurable, and/or a poor prognosis, incurable, palliative, life-limiting, and/or end of life. Processes related to the medical setting include discharge planning, advanced directives, biomedical ethics, and more. Clinical issues are typically noted by a member of the medical team, self-identified by the patient and/or identified during the social worker's assessment. These issues might include the patient's mental health history, suicidality, current exacerbation of emotional or spiritual distress related to previous individual or intergenerational trauma, issues of identity, history of substance use and/or recovery, interpersonal/social dynamics including abuse or violence, economic status and financial hardship, communication with minor children about an adult caregiver's serious illness, legacy work, and finally, issues related to the current sociopolitical environment, namely fear and losses related to the pandemic and emerging protests related to systemic racism and injustice in all aspects of society. All education provided was undergirded by the core ethics and values of the profession of Social Work that serves the inherent dignity of the individual within a complex system (National Association of Social Work Code of Ethics 2017).

In real time, as the pandemic unfolded, areas that required further exploration and learning before the semester concluded were identified by supervisors and presented to interns, individually and in the group setting. The following scenarios serve as prototypes for the modules presented herein, which have been further developed with the intention of guiding their successful implementation in the future. In support of the CSWE core competency to engage diversity and difference in practice (CSWE 2015), supervisors can alter the patient's age, race, culture/ethnicity, gender identity, primary language, abilities/impairments, sexual orientation, or other factors, as needed, to highlight specific learning objectives in the patient scenarios. These modules could easily be adapted to other healthcare settings including but not limited to transplant units, nursing homes, mental health clinics, and substance abuse treatment programs.
Module Example 1: Mid-treatment (Favorable Prognosis)

\section{Scenario A}

Patient, "Jay," is a 21-year-old person of Eastern European descent with testicular cancer $\mathrm{s} / \mathrm{p}$ bilateral orchiectomy with reconstruction who identifies as gender fluid, currently undergoing chemotherapy as an outpatient. Jay broke up with their boyfriend just before receiving the cancer diagnosis. Clinical issues include but are not limited to a history of drug use and recent sobriety, leaving a new job in the fashion industry due to illness/treatment, and navigating boundaries with over-protective parents.

\section{Scenario B}

Patient, "Betty," is a 45-year-old African American woman with breast cancer $\mathrm{s} / \mathrm{p}$ unilateral mastectomy currently undergoing outpatient radiation prior to receiving chemotherapy. Betty is remarried and has a blended family consisting of two children under the age of 10 from her previous marriage and three teenage children from her spouse's previous relationship. The teenagers are beginning to bond with Betty after a fraught beginning. Clinical issues include but are not limited to the death of Betty's mother when Betty was a child and concern around sharing information about her cancer diagnosis and treatment with friends at her church and colleagues at the long-term care facility where she works as an executive nurse leader.

\section{Module Example 2: End of Life (Poor Prognosis, Limited Life Expectancy)}

\section{Scenario A}

Patient, "Sarah," is 48-year-old woman of European descent with end-stage metastatic sarcoma currently admitted to the hospital. Sarah lives with her husband and three minor children who were adopted. Sarah refuses to talk to her minor children about cancer and death. Sarah's husband asked the social worker for assistance with communicating with his children, as he is aware the patient is dying and that the children are afraid to ask questions or upset their mother. Clinical issues include but are not limited to complex medical history and trauma related to infertility, parent-child communication, attachment/bonding concerns with adopted children (abandonment), and legacy work.

\section{Scenario B}

Patient, "Freddy," is a 72-year-old Korean widower, fluent in Korean with limited English proficiency, with widely 
metastatic head and neck cancer. His daughter, Lola, is his healthcare agent. Freddy is hospitalized and does not have decision-making capacity. Lola, and her brother, Junior, disagree about treatment and code status (Do Not Resuscitate). Lola verbalized confusion about her role as healthcare agent and spoke with the social worker about her struggle to make decisions. The medical team requested social work support for the family on issues related to anticipatory grief and advanced directives. Clinical issues include but are not limited to communication barriers, cultural humility, complicated grief and conflict resolution.

\section{Examples of Learning Objectives Covered by the Modules}

\section{Role of Social Worker in the Medical Setting}

Understanding the role of the social worker regarding service to the patient/family in alignment with the NASW Code of Ethics (2017), conducting chart review and writing in the medical record (documentation), clinical assessment, engaging in interdisciplinary collaboration, addressing advanced directives, organizing/facilitating family meetings and interdisciplinary team meetings, understanding and assisting the discharge planning process, requesting ethics consultations.

\section{Clinical Competencies/Modalities}

Conducting biopsychosocial assessments; engaging in active listening; utilizing cognitive behavioral interventions and narrative therapy, among other clinical modalities; clinical engagement and interviewing skills; psychoeducation related to patient's identity, social roles, adjustment to illness (emotional, physical, social, financial, spiritual); understanding advanced directives; teaching communication skills; legacy building, anticipatory bereavement and complicated grief; understanding concepts related to biomedical ethics.

\section{Clinical Issues}

Psychiatric history (suicide assessment and safety planning), substance use, interpersonal conflict/violence, immigration status, spiritual/religious distress/conflict, education level and access to care.

\section{Role-Play Opportunities}

One-on-one with Patient, Couple, Family, Group, member of interdisciplinary team, Ethics consultation.

\section{Intern Assignments}

Reflection papers, Process Recordings, Documentation in the Medical Record, Reading/Viewing (articles, books, movies, videos, webinars, etc.).

\section{Utilizing the Learning Modules in Supervision with Interns}

Supervisors decide which module is most appropriate given interns' clinical skills, experience, and existing knowledge base. Supervisors determine when, in what manner, and what degree of detail to share about cases based on targeted learning goals. For example, interns with less clinical experience might require more background information before engaging in role-play. With more experienced interns, supervisors might choose to provide less background information, anticipating that interns will request additional information related to the role-played interviews. Although the training begins on a basic level, clinically rich interactions should follow with ongoing experiences. Once they have developed a foundation, interns will have opportunities to learn, for example, how to encourage patients to consider different narratives as a therapeutic experience (DeSalvo 1999) within the context of serious illness.

If supervisors use de-identified content from actual cases, less time is needed to prepare for the role-play sessions, interactions are potentially more powerful, and the supervisors provide a more realistic representation of the clinical work. For role-play to be most successful, the supervisors are encouraged to be authentic and to draw from their own personal and professional life experiences. This will help interns understand the patient as a whole person and form a connection with them. The more realistic the encounters, the greater the depth of the interactions. The supervisors' willingness to trust the process, while being unguarded and authentic will impact the quality of the role-playing (Kaushik 2017).

Prior to the role-playing session, supervisors provide interns with a review of the social worker's role, articles related to the educational objectives in advance, the patient's history of presenting illness, some biopsychosocial details, and reason for the referral. Interns are invited to ask questions and request more information. Supervisors teach interns how to initiate a session with a patient considering the following factors: where is the patient located (in clinic, in the hospital); who else is in the room; how should interns enter and introduce themselves; is this the first encounter with the patient; how would interns initially engage with patients? After this review, the one-on-one role-play between supervisor and intern begins with the understanding that either supervisor or intern could pause the interaction to ask questions, indicate that guidance is 
required, make supervisory suggestions, or comment on successes, thereby building the intern's confidence. The intern learns and practices how to ask open-ended, exploratory, and follow-up questions. The supervisor provides the intern with guidance related to asking questions that unpack what the patient understands about their disease or other concerns, framing how to inquire about challenging and perhaps intimate topics, interpreting silences and using silence (Hill et al. 2003), and determining possible responses during the clinical encounter. Supervision also focuses on demonstrating empathy and bearing witness to patient experiences while reviewing, for example, the use of exploratory questions and deliberate silences.

Role-playing can be used with a group of interns, as well as with individuals. The presentation of a family system and opportunity for a family counseling session, or complicated team meeting, can be role-played by several interns. After multiple interactions, interns begin to trust their clinical decision making and may become more comfortable with interactions with one or multiple participants. With more clinical opportunities, role-play interactions will likely become more nuanced and meaningful. As interns' knowledge base expands and their interviewing skills become more sophisticated, they will have opportunities to learn how they might intervene and support patients and families within the medical setting.

Finally, exploring interns' self-awareness and use of self throughout the training is an essential component of learning how to engage in clinical work. Reflective practice and using one's own emotional responses, for both supervisors and interns, during clinical encounters is key to the therapeutic process (Kaushik 2017). Supervision related to nuanced clinical work will be well-served when supervisors are willing to skillfully reveal authentic responses and feelings.

\section{Individual and Group Supervision During the Pandemic}

As a result of the transition to virtual learning, interns reported feeling disconnected from the hospital setting, from their professional responsibilities, and from their clinical work. Supervisors were mindful of the psychological and emotional disturbance triggered by the pandemic for both staff and interns, and a trauma-informed approach was integrated in the redesign of the program (CSWE 2018). Individual and group supervision continued weekly and provided continuity and opportunities to discuss the overlap of their field placement and the pandemic. Supervisors provided updates from administration to help interns understand how the hospital environment and patient care shifted since they left the hospital. Supervisors encouraged interns to reflect on issues and themes that could arise if they were still working at the hospital. Supervision focused on the parallel experience of managing existential uncertainty and mortality awareness, navigating professional and personal identities, and engaging in termination.

Throughout the academic year, supervision focused on developing interns' critical thinking related to common themes that patients cope with, such as uncertainty about the future, unexpected adjustments to lifestyle and routine, and ongoing health anxiety. Supervision enabled interns to process their own emotional response that was evoked during their work with patients, which is an important component of the training offered at the cancer center. When the pandemic occurred, many existential themes that were present in the daily work for interns were brought to the forefront of society. Although interns had been processing their own death anxiety throughout the internship, they appeared to experience it somewhat differently during the pandemic. Earlier in the year, the parallel experience of confronting their own mortality while working with their patients was gradual. At times, interns had a choice of how much or little to engage with the content. During the pandemic, end-oflife issues became intensely present in their personal and academic lives so that it became impossible to avoid this topic. Trauma-informed supervision (CSWE 2018) focused on helping interns process the accelerated pace in which they dealt with mortality awareness for themselves and for their loved ones. Discussion highlighted how interns can use their own experience of these issues to better understand the experience of their patients. Supervisors also identified the parallel experience of uncertainty and intensified vulnerability occurring between supervisors and interns, as well as between social workers and patients.

As interns reflected on how the pandemic may have altered their field placement, themes emerged from the CSWE core competencies, such as ethical and professional behavior and advancing human rights and social, economic and environmental justice (CSWE 2015). During supervision, interns discussed how to navigate the overlap of one's professional and personal identities in this context. The group supervisor facilitated discussion about the potential challenges associated with caring for the psychosocial needs of patients while also coping with the personal impact of the pandemic. Interns imagined that it would be more difficult for them emotionally to support patients who were facing death while being confronted by the potential for their own death due to the pandemic. Supervision encouraged students to process how they might handle a shift in boundaries as the emotional experience of their patients mirrored aspects of their own, leading to a rich conversation about the use of self (Kaushik 2017). The group supervisor also facilitated discussion about how privilege and aspects of one's identity may profoundly alter a person's experience of the pandemic; issues related to race, socioeconomic status, and geographic region were explored. 
Another theme that emerged was the challenge of discerning between professional and personal roles as interns interacted with people in their personal lives. Interns spoke about offering support to friends and family, without the self-imposed pressure of using the skills they had acquired to "be a social worker." Interns acknowledged that in some instances their social work training helped them have difficult conversations with loved ones. For example, interns discussed how they were able to use their knowledge of advanced care planning (i.e. appointing a healthcare agent, completing advance directives) to initiate discussions with their family members on this topic during the pandemic. They shared that the internship gave them the language and skillset to approach these sensitive discussions in their personal lives. Interns were also mindful of how they might respond to loved ones who were directly impacted by COVID-19. Interns contemplated the possibility of experiencing pressure to respond "perfectly" because this was their area of expertise. They acknowledged that this pressure might prevent them from bringing their own humanity to these interactions because they were placing an emphasis on crafting a clinical response, rather than responding in a familiar and genuine way. Supervisors encouraged interns to explore the blending of their professional and personal identities in order to find a balance that was authentic. Time was spent processing commonalities and distinctions between their professional and personal identities.

Another component of supervision focused on the termination process, which was altered in important ways as a result of the pandemic. Due to the nature of the social work role at the cancer center, interns engaged in termination at varying points throughout the academic year. Depending on the inpatient unit or outpatient clinic that the interns were training in, some interns encountered termination daily due to one-time clinical interactions. The relationships between patients and interns were sometimes unexpectedly terminated due to patient deaths or accelerated due to patients being discharged from the hospital. Some interns who met with patients over a longer period were able to prepare for and discuss termination in a more structured and processoriented way. Throughout the academic year, discussion focused on clinical themes that arise during termination, such as the emotional response triggered by separation and loss at the end of a patient relationship. Siebold (1991) discusses the process of anticipatory grief before the end of a patient relationship, and how the period prior to termination can provide valuable opportunities for social workers and patients to process the impact of loss. Ambivalence related to the unexpected end of patient care due to the pandemic, the lack of closure, and an inability to engage in thoughtful termination with some patients was discussed. Supervision focused on allowing interns to process their own emotional reaction to this separation and loss.
The transition to virtual learning during the pandemic drastically shifted the way in which interns terminated with their supervisors, with the hospital, and with each other. Virtual learning became essential in facilitating discussion related to the end of these relationships. Interns were invited to process their growth and development over the course of their time together. Supervision also focused on processing the emotion associated with the end of their field placement. Again, interns demonstrated the self-awareness that was cultivated throughout the internship to reflect on the resolution of their internship experience. Disappointment and sadness related to the way in which the internship ended was validated. Finally, interns discussed the gratitude they felt for the opportunities they experienced throughout the year and for the ability to continue their internship virtually during such an uncertain time.

\section{Overall Benefits \& Future Considerations}

The virtual platform is a necessary component for delivering healthcare during and after the COVID-19 pandemic. This creative adaptation of field instruction can be used to enhance the experience for interns in a variety of healthcare settings moving forward. This adjustment to the existing program provided interns with the opportunity to develop skills necessary to conduct meaningful clinical work without direct patient contact. Direct in-person interaction is the preferred method for clinical training; however, virtual programming can be used successfully with interns to develop their skills. Supervisors were also required to be flexible and develop their abilities and comfort using this format. Targeted learning was thoughtfully prepared and helped to increase interns' confidence, understand core competencies, and cultivate their clinical proficiency. Another benefit of virtual programming is that it can accommodate diverse learning styles. In addition, supervisors may determine that interns are not yet ready for direct interactions with patients. This format gives interns the opportunity to practice and develop their interpersonal skills before engaging patients /clients.

A virtual program may be more geographically inclusive and will serve as a complement to in-person, on-site experiences. Shifting to the virtual format enables interns who may be placed at various locations within an organization to have a more consistent and common experience with their peers. Moving forward, Schools of Social Work will likely modify field placement requirements to include virtual platforms and telehealth. This innovative hybrid of traditional in-person and virtual training can serve as a prototype for future educational programming. Were it not for the pandemic, this instructive and strengths-based model may have been years away. 
It is important to acknowledge the limitations associated with virtual learning for social work graduate students. While advances in technology have made this practice possible, virtual learning is not always optimal. Some interns may not have access to reliable technology. In addition, virtual learning requires interns to identify a private and quiet space that is conducive to online instruction; however, that may not be possible due to various factors. Patient confidentiality and one's readiness to be vulnerable during supervision are significant concerns if interns are not able to find an appropriate workspace. Schools of Social Work are likely to provide additional resources to facilitate virtual learning, including how to manage the isolation that may arise while working remotely.

Another potential consideration is the blurring of professional and personal identities, which is as relevant for supervisors as it is for interns. Supervisors may notice that it feels more revealing to participate in videoconferencing or phone calls from their homes. With the virtual format, both interns and supervisors may have access to one another's private life, which can challenge boundaries in the internsupervisor relationship (Knapp and Slattery 2004). This is an opportunity for supervisors to reflect on the balance of this exposure and their role as experts who possess knowledge and authority.

In the future, the newest graduates from social work masters' programs will likely have had less field experience due to the ongoing social distancing guidelines required to maintain public safety during this unprecedented public health crisis. This virtual program, combined with on-site training, will provide an increased opportunity for developing clinical skills and understanding professional responsibilities in the healthcare setting. The virtual model can also be helpful for onboarding new social work staff who may be working remotely, due to the pandemic and the shift to telehealth.

Using this model, interns reported a similar level of skill development as compared to their in-person experiences. Supervisors observed a comparable and, in some cases, enhanced level of clinical skill acquisition through role-play. Field placements that utilize a virtual approach might consider implementing an evaluative process to ensure that the program meets the educational objectives and is a satisfying and enriching experience for the interns. Evaluative methods for the interns might include non-graded assignments, such as process recordings, journal writing and/or reflection papers, midterm and final evaluations, feedback from supervisors and other interns from the cohort (Molina et al. 2018). Feedback on training content and the experience with field instructors and supervisors may be gathered from the interns through individual supervision, group supervision, and meetings with the educational coordinator. The efficacy of this virtual approach should continue to be evaluated and adjusted over time.
The impact of the pandemic, in all aspects of society, will be ongoing. As a result, educational programs for future social workers will need to prepare interns through virtual platforms for becoming part of the frontline workforce. It is recommended that training be provided in trauma work, ambiguous loss and complicated grief, suicide assessment, interpersonal violence and substance misuse, racial bias and disparities in healthcare, and the distress, moral injury and ethical challenges that impact healthcare workers. This enhanced social work training related to the pandemic is relevant for Clinical, Administrative, Policy, and Community Organizing tracks.

\section{Conclusion}

The COVID-19 pandemic caused an abrupt conclusion of the on-site field placement for social work interns who could not work remotely. Virtual programming became necessary to meet the interns' remaining educational requirements through a patient-less learning experience. This change provided an opportunity for enhanced learning and proper termination from the program. Interns expressed satisfaction with the ongoing learning opportunities and appreciation for the genuine care that was extended to them during this unprecedented health crisis. This remote/virtual adaptation to the internship serves as a prototype for future programs that aim to include innovative strategies to meet the diverse learning needs of individual students and enrich clinical supervision that could be used in a broad range of healthcare settings.

Acknowledgement The authors would like to thank Penny Damaskos, PhD, LCSW-R, OSW-C and Anne Martin, PhD, LCSW-R for their guidance and support.

\section{References}

Centers for Disease Control and Prevention. (2020). Clinical Care Guidance for Healthcare professionals about Coronavirus (COVID-19). Retrieved June 10, 2020 from https://www.cdc.gov/ coronavirus/2019-ncov/hcp/clinical-care.html.

Centers for Disease Control and Prevention. (Updated June 2, 2020). Interim clinical guidance for management of patients with confirmed coronavirus disease (COVID-19). Retrieved June 10, 2020 from https://www.cdc.gov/coronavirus/2019-ncov/hcp/clinicalguidance-management-patients.html.

Centers for Disease Control and Prevention. (Updated May 26, 2020). Outpatient and ambulatory care settings: Responding to community transmission of COVID-19 in the United States. Retrieved June 10, 2020 from https://www.cdc.gov/coronavirus/2019-ncov/ hcp/ambulatory-care-settings.html.

Council on Social Work Education. (Updated May 9, 2020). CSWE and Commission on Accreditation Statement. Retrieved June 10, 2020 from https://www.cswe.org/CSWE/media/AccredidationPD Fs/COA-Field-Reduction-Update-05-09-2020.pdf. 
Council on Social Work Education. (2018). Specialized practice curricular guide for trauma informed social work practice. Retrieved Sept 14, 2020 from https://www.cswe.org/Education-Resou rces/2015-CurricularGuides/2015EPAS_TraumaInformedS Wweb-(2).aspx.

Council on Social Work Education. (2015). Educational policy and accreditation standards for baccalaureate and master's social work programs. Retrieved Sept 15, 2020 from https://www.cswe. org/getattachment/Accreditation/Standards-and-Policies/2015EPAS/2015EPASandGlossary.pdf.aspx.

Department of Health \& Human Services. (March 2020). COVID$19 \&$ HIPAA Bulletin: Limited waiver of HIPAA sanctions and penalties during a nationwide public health emergency. Retrieved June 10, 2020 from https://www.hhs.gov/sites/default/files/hipaa -and-covid-19-limited-hipaa-waiver-bulletin-508.pdf.

DeSalvo, L. (1999). Writing as a way of healing: How telling our stories transforms our lives. Boston: Beacon Press.

Hill, C. E., Thompson, B. J., \& Ladany, N. (2003). Therapist use of silence in therapy: A survey. Journal of Clinical Psychology, 59(4), 513-524.

Hogan, B., Marsh, J. \& Golding, B. (April 8, 2020). Cuomo: New York State is 'flattening the curve' of coronavirus cases. NY Post. Retrieved June 10, 2020 from https://nypost.com/2020/04/08/newyork-state-is-flattening-the-curve-of-coronavirus-cases-cuomo/.

Kaushik, A. (2017). Use of self in social work: Rhetoric or reality. Journal of Social Work Ethics and Values, 14(1), 21-29.

Knapp, S., \& Slattery, J. M. (2004). Professional Psychology: Research and Practice, 35(5), 553-558.

Molina, V., Molina-Moore, T., Smith, M., \& Pratt, F. (2018). Bridging education and practice with a competency-based learning contract. Journal of Teaching in Social Work, 38(1), 18-27.

National Association of Social Work. (2017). NASW Code of Ethics. Retrieved June 10, 2020 from https://www.socialworkers.org/ about/ethics/code-of-ethics.
New York Family Health Care Decisions Act (FHCDA) (Chapter 8 of the Laws of 2010, adding Public Health Law Ch. 29-CC and 29-CCC).

Siebold, C. (1991). Termination: When the therapist leaves. Clinical Social Work Journal, 19(2), 191-204.

Publisher's Note Springer Nature remains neutral with regard to jurisdictional claims in published maps and institutional affiliations.

Barbara Mitchell is a clinical social worker at Memorial Sloan Kettering Cancer Center in New York, NY. They provide psychosocial counseling to cancer patients and families in inpatient and outpatient settings. Interventions focus on adjustment to diagnosis and treatment, survivorship, end of life care, and bereavement.

David Sarfati is a senior clinical social worker at Memorial Sloan Kettering Cancer Center in New York, NY. They provide psychosocial counseling to cancer patients and families in inpatient and outpatient settings. Interventions focus on adjustment to diagnosis and treatment, survivorship, end of life care, and bereavement.

Melissa Stewart is a senior clinical social worker at Memorial Sloan Kettering Cancer Center in New York, NY. They provide psychosocial counseling to cancer patients and families in inpatient and outpatient settings. Interventions focus on adjustment to diagnosis and treatment, survivorship, end of life care, and bereavement. She is also an interfaith/interspiritual minister. 